\title{
Alter
}

Revue de phénoménologie

26 | 2018

La nature

\section{La nature entre phénomène et spéculation}

\section{Inga Römer}

\section{OpenEdition}

Journals

Édition électronique

URL : http://journals.openedition.org/alter/545

DOI : $10.4000 /$ alter.545

ISSN : 2558-7927

\section{Éditeur :}

Association ALTER, Archives Husserl (CNRS-UMR 8547)

\section{Édition imprimée}

Date de publication : 31 décembre 2018

Pagination : 15-29

ISBN : 978-2-9550449-4-0

ISSN : 1249-8947

\section{Référence électronique}

Inga Römer, « La nature entre phénomène et spéculation », Alter [En ligne], 26 | 2018, mis en ligne le 31 décembre 2019, consulté le 22 mars 2020. URL : http://journals.openedition.org/alter/545 ; DOI :

https://doi.org/10.4000/alter.545 


\section{LA NATURE ENTRE PHÉNOMÈNE ET SPÉCULATION}

Inga Römer

Pour paraphraser une célèbre formule de Heidegger, l'on pourrait dire que la nature appartient à ce qui nous est le plus proche, et en même temps, qu'elle demeure parmi ce qui nous est le plus éloigné1. Cette situation paradoxale relève semble-t-il d'une tension constitutive : d'un côté, la nature est, par rapport à nous, en vis-à-vis, et peut ainsi devenir l'objet de notre analyse ; d'un autre côté, nous sommes nous-mêmes des êtres naturels, qui, en tant qu'êtres pensants, sommes enracinés dans la nature. Ainsi, la nature se trouve-t-elle aussi bien « devant» nous que "derrière, et en nous ». Et dans la phénoménologie de tradition husserlienne, c'est ce deuxième aspect qui pose les problèmes les plus fondamentaux : comment est-il possible de s'interroger sur la nature en tant que " phénomène ", si la nature comme telle dépasse le champ du phénoménalisable? Est-il alors nécessaire de faire un pas vers la spéculation pour rattraper ce qui, par principe, échappe à la phénoménalité ?

Ce problème, toutefois, ne concerne pas uniquement la phénoménologie, mais surgit déjà dans l'espace de tension entre Kant et $l^{\prime}$ «idéalisme allemand ». Afin d'en préciser les coordonnées, c'est dans un tel espace que nous déploierons nos premières réflexions, avant de discuter l'argument spéculatif par lequel Quentin Meillassoux entend les déplacer puis, sur le fondement des critiques que nous tenterons de lui adresser, nous nous tournerons vers la phénoménologie de Husserl, Heidegger, Ricœur et Levinas, afin d'esquisser quelques perspectives sur une possible phénoménologie de la nature articulant phénomène

1 Voir M. Heidegger, Sein und Zeit, F. W. von Herrmann (éd.), Gesamtausgabe. I. Abteilung : Veröffentlichte Schriften 1910-1976, vol. 2, Francfort-sur-le-Main, Vittorio Klostermann, 1977, §5. 
et spéculation. Nous terminerons avec l'esquisse d'une conception temporelle de la nature.

\title{
I. La nature en tant que «totalité des phénomènes »- l'idée kantienne à l'épreuve
}

$\mathrm{Au}$ début de la préface des Principes métaphysiques de la science de la nature, Kant définit d'une manière concise ce qu'il entend par « nature » :

\begin{abstract}
Si l'on prend le mot de nature simplement au sens formel, où il signifie le premier principe interne de tout ce qui appartient à l'existence d'une chose, il peut alors y avoir autant de sciences de la nature qu'il y a de choses spécifiquement différentes, chacune devant contenir son propre principe interne des déterminations qui appartiennent à son existence. Mais la nature est aussi prise au sens matériel, non comme une propriété constitutive, mais comme l'ensemble de toutes les choses en tant qu'elles peuvent être des objets de nos sens, et par suite aussi des objets d'expérience, de sorte que l'on comprend sous ce mot la totalité des phénomènes, c'est-à-dire le monde sensible, en excluant tous les objets non sensibles. La nature, prise en ce sens du mot, comporte alors, conformément à la distinction principale de nos sens, deux grandes parties, l'une contenant les objets des sens externes, et l'autre contenant l'objet du sens interne, de sorte qu'une double doctrine de la nature est possible, la "doctrine des corps » et la "doctrine de l'âme ", où la première considère la nature étendue, et la seconde la nature pensante ${ }^{2}$.
\end{abstract}

Retenons de ce passage trois traits principaux. Premièrement, «nature », en un sens formel, signifie pour Kant « le premier principe interne de tout ce qui appartient à l'existence d'une chose ", tandis que la catégorie de l'existence n'a de sens pour nous que par rapport aux formes de notre sensibilité. Deuxièmement, "nature » au sens matériel signifie « l'ensemble de toutes les choses en tant qu'elles peuvent être des objets de nos sens », ou encore « la totalité des phénomènes » ou « le monde sensible». Et troisièmement, il s'agirait de distinguer deux théories possibles de la nature, l'une du corps et l'autre de l'âme, et ceci relativement à nos deux "sens", le sens externe et le sens interne même si l'impossibilité d'appliquer la mathématique aux phénomènes du sens interne conduit finalement Kant à exclure la possibilité d'une telle science de l'âme ${ }^{3}$. Or si la nature est bien, comme ensemble des

\footnotetext{
2 I. Kant, Metaphysische Anfangsgründe der Naturwissenschaften, Ak. IV, Berlin, De Gruyter, 1968, p. 465-565, ici p. 467 (Principes métaphysiques de la science de la nature, trad. A. Pelletier, Paris, Vrin, 2017).

${ }^{3}$ Voir Ak. IV, p. 471.
} 
phénomènes en tant qu'ils sont structurés par des lois, ce qui se trouve « devant» nous, comment rendre compte de la «nature » de cet être pour qui elle est phénomène?

Kant semble toucher à ce problème dans la préface à la première édition de la Critique de la raison pure, où il souligne le statut secondaire de la partie subjective de la déduction transcendantale par rapport à sa partie objective. "La question capitale reste toujours de savoir : que peuvent connaître, et jusqu'où, l'entendement et la raison, libres de toute expérience, et non : Comment est possible le pouvoir de penser luimême ? ». Et il ajoute : "cette dernière question est en quelque sorte la recherche de la cause pour un effet donné, et contient dans cette mesure quelque chose de semblable à une hypothèse (bien que [...] il $n^{\prime}$ en aille pas en fait ainsi) $»^{4}$. Dans ce passage difficile, Kant semble de nouveau dire deux choses. D'une part, si la question de savoir ce que «peuvent connaître l'entendement et la raison, libres de toute expérience empirique a posteriori » est la «question capitale », c'est parce qu'elle semble ouvrir un questionnement métaphysique. Le fait qu'il dépende de nos facultés de connaissance pourrait certes nous orienter vers l'idée selon laquelle une telle «question capitale » serait celle du pouvoir de penser et de sa possibilité - et c'est justement contre cette confusion que Kant entend nous mettre en garde. Pourtant, et en second lieu, nous pouvons et devons aussi poser la question de la possibilité même du pouvoir de penser, et chercher "en quelque sorte » une «cause » pour ce pouvoir effectif. Ce « en quelque sorte » fait alors signe vers une deuxième confusion à prévenir : on pourrait prendre cette recherche de la "possibilité » pour celle d'une cause naturelle du penser. Or une cause naturelle en tant que telle peut toujours être révisée, n'est jamais absolument certaine, et ce qu'il s'agit ici d'exhiber, ce n'est précisément pas une cause naturelle hypothétique mais une raison a priori et nécessaire qui s'appuie sur les conditions de la possibilité de l'expérience. En ce sens, la prétendue question de l'enracinement naturel du pouvoir de penser se trouve ramenée à la question de la possibilité a priori de l'expérience - ou pour le dire autrement: la question de la cause naturelle du pouvoir de penser compris comme fait empirique se trouve d'abord reconduit à celle de la validité de la catégorie de la causalité, laquelle trouve sa réponse dans une raison a priori s'appuyant sur la possibilité de l'expérience. Aussi la tentative d'interroger la nature «derrière et en nous » se

4 I. Kant, Kritik der reinen Vernunft, Hambourg, Meiner, A XVII (Critique de la raison pure, trad. A. J.-L. Delamarre et F. Marty à partir de la traduction de J. Barni, Paris, Gallimard, 1980). 
trouve-t-elle réduite à une saisie de la nature «devant nous », de la nature en tant que phénomène.

Ce refus kantien de reconduire le pouvoir de penser, y compris les formes de la sensibilité et les catégories de l'entendement, à une causalité de la nature, nous confronte donc à une alternative : ou bien rester fidèle à la philosophie transcendantale kantienne qui lie les structures fondamentales de la nature à la possibilité de l'expérience et situe la question de l'engendrement naturel de nos facultés sur un plan dérivé, ou bien tenter de penser directement une nature capable d'engendrer nos facultés - formes de la sensibilité et catégories de l'entendement -, mais au prix d'un changement radical de contexte d'analyse.

Or telle est justement la voie suivie par le jeune Schelling. Dans son Système de l'idéalisme transcendantal de 1800, souvent considéré comme l'achèvement de la première phase de sa pensée avant qu'elle ne devienne " philosophie de l'identité », il tente ainsi de faire droit à une complémentarité entre la philosophie de la nature et la philosophie transcendantale - $d$ 'une manière dont nous résumerons ici encore les idées principales. Partons de la proposition suivante: "Tout savoir repose sur l'accord (Übereinstimmung) d'un objectif avec un subjectif. Car on ne sait que le vrai, et la vérité se trouve dans l'accord des représentations avec leurs objets $»^{5}$. Le fait même du savoir présuppose $q u^{\prime}$ il existe un tel accord, et le problème ne peut consister qu'à chercher comment le penser. Or il y a, d'après Schelling, deux possibilités et deux seulement: " ou c'est l'objectif qui est supposé l'élément premier, et alors la question est celle-ci : comment se fait-il qu'un subjectif vienne s'accorder à lui ? »; ou « le subjectif est supposé l'élément premier » et « le problème est de savoir comment il se fait qu'un objectif s'y ajoute et $s^{\prime}$ accorde à lui $»^{6}$. Or pour Schelling, « l'ensemble de tout ce qui est purement objectif dans notre savoir » est la "nature», et «l'ensemble de tout ce qui est subjectif » est « le moi, ou l'intelligence » ${ }^{7}$. L'alternative est donc la suivante : comprendre l'accord à partir de la nature, ou bien le comprendre à partir de l'intelligence. Or dans son œuvre de 1800, $c^{\prime}$ est à un " parallélisme de la nature avec l'intelligence $»^{8}$ que tente de se ranger Schelling, lequel ne saurait être présenté (dargestellt) par la philosophie de la nature seule ou par la philosophie transcendantale seule mais exige leur complémentarité : il faut « ces deux sciences, qui,

\footnotetext{
${ }^{5}$ F. W. J. Schelling, System des transzendentalen Idealismus, Ausgewählte Schriften, vol.1, Francfort-sur-le-Main, Suhrkamp, 1985, p. 395-702, ici p. 407 (Système de l'idéalisme transcendental, trad. P. Grimblot, Paris, Librairie philosophique de Larange, 1842, p. 2, traduction modifiée).

${ }^{6}$ Ibid., p. 408 , p. 409 (p. 2, p. 4, traduction modifié).

${ }^{7}$ Ibid., p. 407 (p. 1, traduction modifiée).

8 Ibid., p. 399 (p. LXVIII).
} 
précisément pour ce motif, doivent être les deux sciences éternellement opposées, et ne peuvent jamais être réunies en une seule science ${ }^{9}$.

Aussi, la science de la nature a pour « tendance nécessaire [...] d'arriver de la nature, comme point de départ, à la sphère intellectuelle »10, et ainsi de répondre à la question : "Comment à la nature s'ajoute-t-il l'intelligent, ou en d'autres termes : comment la nature arrive-t-elle à être représentée ? ${ }^{11}$. L'achèvement le plus élevé de la science de la nature serait alors atteint «si l'on parvenait à faire entrer complètement toutes les lois de la nature dans la sphère purement intellectuelle, où résident les lois de l'intuition et de la pensée [die vollkommene Vergeistigung aller Naturgesetze zu Gesetzen des Anschauens und des Denkens] »12. Ici, "[1]es phénomènes (le matériel) doivent s'évanouir entièrement, et les lois (le formel) finissent par demeurer seules $»^{13}$. En ce sens, "[1]a nature se résoudrait (sich auflöste) dans l'élément intellectuel ${ }^{14}$, elle se résorberait « objectivement » dans l'homme et retournerait ainsi entièrement à soi-même. De sorte que lorsque l'homme achève la science de la nature, il exhibe le fait que la nature est " originairement identique » avec l'intelligence et ce qui en nous est conscient. La philosophie transcendantale - la seule qui sera développée dans le Système de l'idéalisme transcendantal - procède pour sa part de manière inverse. Elle part « du subjectif, comme premier et absolu, et en [fait] sortir l'objectif $\gg^{15}$. À partir de ce subjectif premier, elle doit montrer en quel sens la certitude des choses hors de nous est un préjugé nécessaire, et comment nous arrivons nécessairement à la conception d'une objectivité de la nature hors de nous. De fait, ces deux sciences présupposent nécessairement une "harmonie préétablie » entre le monde idéel et le monde réel, comme le dit Schelling avec une expression leibnizienne. Et « on ne peut concevoir cette harmonie préétablie, que si l'activité qui produit le monde objectif est originairement identique avec celle qui se manifeste dans le vouloir, et vice versa $»^{16}$. La présupposition est alors plus précisément celle d'une identité entre l'activité qui engendre la nature et l'activité du moi et de son vouloir. Et les deux sciences ont alors pour tâche de montrer, avec le plus de lucidité possible, le «que » et le «comment» de cette identité.

\footnotetext{
${ }^{9}$ Idem.

${ }^{10}$ Ibid., p. 408 (p.3).

${ }^{11}$ Ibid., p. 408 (p. 2, traduction modifiée).

12 Ibid., p. 408 (p. 3, nous ajoutons l'original).

${ }^{13}$ Ibid., p. 408 (p. 3, traduction modifiée).

14 Ibid., p. 409 (p. 4$)$.

${ }^{15}$ Ibid., p. 410 (p. 5, traduction modifiée).

${ }^{16}$ Ibid., p. 416 (p. 13, traduction modifiée).
} 
Il semble qu'avec le Schelling de 1800, nous ayons ainsi rejoint le second versant du dilemme plus haut présenté. La nature n'est pas réduite à un phénomène " pour et devant nous ", comme chez Kant, mais s'avère tout aussi bien "derrière et en nous ». La difficulté, néanmoins, est que le raisonnement de Schelling est alors nécessairement circulaire : une présupposition nécessaire pour tout savoir, celle de l'identité entre l'intelligence et la nature, en constitue le point de départ, avant que le double mouvement de la nature à l'intelligence et de l'intelligence à la nature n'exhibe le «que » et le «comment » de cette identité. Sans cette présupposition spéculative, il n'y aurait ni savoir ni philosophie.

\section{Le saut spéculatif de Quentin Meillassoux}

L'un des défis actuels pour la tradition philosophique qui, s'initiant avec Kant, passe par l'idéalisme allemand et se développe jusqu'à la phénoménologie et à la philosophie analytique du langage, est ledit " réalisme spéculatif » dont le propre est de chercher une issue à ce qu'il nomme le « corrélationisme ». Or, ce défi « réaliste » étant essentiellement lié au problème de la nature, nous nous proposons maintenant d'analyser ce qui nous semble en constituer les deux arguments centraux. Y a-t-il donc, dans d'Après la finitude ${ }^{17}$, une solution au dilemme constitutif de la philosophie de la nature?

Le premier argument central de Meillassoux est négatif, et part du problème de « l' ancestral » : il serait impossible à la tradition « corrélationiste » de Kant jusqu'à la phénoménologie de rendre compte d'une réalité antérieure à l'existence des êtres humains, de leur conscience et de leur pensée, alors qu'il appartiendrait aux sciences, dans leur pratique effective, de conclure à une telle réalité à partir des matériaux archifossiles. Et c'est à cet égard que la thèse centrale du corrélationisme serait porteuse $d^{\prime}$ " un véritable contre-sens $»^{18}$. Avant de mettre à l'épreuve cet argument, tournons-nous vers son versant positif, relatif à la nécessité d'un dépassement de la corrélation de l'intérieur et fondé sur une conception de la facticité, non plus comme une limite pour notre pensée mais comme principe de l'absolu et ainsi de la réalité en soi ${ }^{19}$. L'argument est in nuce le suivant : pour le corrélationiste, sa facticité consiste en ce que son existence n'est pas nécessaire, en ce qu'il

\footnotetext{
${ }^{17}$ Q. Meillassoux, Après la finitude. Essai sur la nécessité de la contingence, Paris, Seuil, 2006.

${ }^{18}$ Ibid., p. 35.

${ }^{19}$ Voir le chapitre « Le principe de factualité », op. cit., p. 81-121.
} 
pourrait ne pas être ; la possibilité de sa propre non-existence ne peut pourtant pas être conçue comme un simple corrélat de sa pensée, puisqu'elle concerne la possibilité du non-être de son propre être pensant ; Meillassoux en déduit que le corrélationiste ne peut penser sa propre non-existence que s'il comprend sa propre facticité comme principe d'un en-soi, indépendant de la pensée ; la contingence universelle serait ainsi la seule nécessité absolue, et de cette proposition s'ensuivrait le matérialisme spéculatif de l'hyper-chaos défendu par Meillassoux. Cet argument nous fait-il sortir du " corrélationisme »? À vrai dire, nous y diagnostiquons un saut spéculatif problématique, consistant à déduire de la simple facticité du cogito performatif le principe de la factualité en soi, et ainsi à convertir un moment de la facticité, l'impossibilité d'en donner une raison suffisante, en un principe positif de l'absolu. Or c'est confondre deux moments de la facticité : de la facticité comme impossibilité de donner une raison pour l'existence métaphysique du cogito, Meillassoux conclut que la facticité comme être-jeté est un être-jeté dans un cosmos qui est lui-même positivement caractérisé par le chaos et la contingence totale. Mais cela, nous semble-t-il, est un non sequitur : en tant qu'ego je suis certes jeté au sein de quelque chose que je ne connais pas et dans lequel il me faut trouver une orientation, ce que je ne trouverai jamais entièrement. Mais de cela, on ne saurait nullement déduire que ce en quoi je suis jeté serait positivement caractérisé par un hyper-chaos en soi.

Résumons donc cette première partie de nos réflexions : chez Kant, la nature n'est interrogée qu'en tant que phénomène, et cette limite ne se trouve dépassée par Schelling qu'au prix de la présupposition spéculative d'une identité de la nature et de l'intelligence ; quant à Meillassoux, qui entend congédier le cercle corrélationiste, il recourt luimême à un saut spéculatif consistant à faire de la simple facticité de l'ego un principe positif du cosmos lui-même. Le dilemme de la nature entre phénomène et spéculation demeure donc intact : ou bien nous nous contentons d'une analyse de la nature en tant que phénomène "devant nous », sans poser la question de la naturalité de celui pour qui la nature est phénomène, ou bien nous nous proposons de penser également une nature " derrière et en nous ", mais nous semblons alors condamnés à quitter le plan de la phénoménalité en faveur d'une présupposition ou même d'un saut spéculatif.

Il est donc temps de nous tourner vers la phénoménologie: la phénoménologie demeure-t-elle du côté kantien du dilemme en faisant de la nature un simple phénomène ? Dispose-t-elle de moyens " spéculatifs » spécifiques qui lui permettraient d'en habiter l'autre versant? 
Quelles ressources a-t-elle pour traiter et peut-être résoudre le dilemme en question?

\section{Le retrait dans la phénoménalité - perspectives phénoménologiques sur la nature}

L'on trouve dans la tradition phénoménologique une figure de pensée qui semble incarner cet entre-deux de la phénoménalité et de la spéculation : celle d'un retrait dans la phénoménalité - l'idée selon laquelle quelque chose dans la phénoménalité même s'annonce comme se retirant ${ }^{20}$. Cette figure de pensée, avec ses différentes concrétisations - nous insisterons ici sur la trace et la résistance - pourrait-elle s'avérer utile pour aborder le dilemme de la nature? Nous nous contenterons ici d'esquisser quelques lignes d'une réponse affirmative à cette question, en insistant sur la fonction qu'y occupe le problème de la temporalité.

\section{Husserl - la constitution rétrospective de la nature avant la conscience}

Dans un passage célèbre des Ideen I, Husserl développe la thèse selon laquelle toute chose se donne par esquisses et sous un horizon d'indétermination déterminable, et précise que « [n]ul Dieu ne peut y changer quoi que ce soit $»^{21}$. Plus radical que Kant, Husserl clôt donc la possibilité $\mathrm{d}^{\prime}$ une autre manière de saisir la chose que celle que nous connaissons - puisqu'une donation sans esquisses la résorberait dans le flux de la conscience et en ferait un moment réel de ce flux. En ce sens, la chose en tant que telle est la chose en tant que phénomène pour nous, donnée en esquisses avec un horizon ouvert. Mais cette radicalisation nous confronte à une difficulté - celle-là même que pointe Meillassoux en parlant d'une contradiction du corrélationisme : si la chose en tant que chose est la chose pour notre conscience,

\footnotetext{
${ }^{20}$ Heidegger parle d'une "Phänomenologie des Unscheinbaren», d'une "phénoménologie de l'inapparent » dans le séminaire de Zähringen, une formule qui est devenue célèbre notamment en France. M. Heidegger, «Seminar in Zähringen 1973 », in Seminare, C. Ochwadt (éd.), Gesamtausgabe vol. 15, Francfort-sur-le-Main, Vittorio Klostermann, 1986, p. 372-400, ici p. 399 («Le séminaire de Zähringen », in Questions III et IV, Paris, Gallimard, « tel », 1976, p. 459-488, ici p. 487). ${ }^{21}$ E. Husserl, Ideen zu einer reinen Phänomenologie und phänomenologischen Philosophie. Allgemeine Einführung in die reine Phänomenologie, K. Schuhmann (éd.), Husserliana vol. III/1, La Haye, Martinus Nijhoff, 1976, p. 92 (Idées directrices pour une phénoménologie et une philosophie phénoménologiques pures. Introduction générale à la phénoménologie pure, trad. P. Ricœur, Paris, Gallimard, 1950, p. 142).
} 
comment rendre compte des choses avant l'existence de cette conscience?

À vrai dire, Husserl n'a pas ignoré cette question, et il y revient à plusieurs reprises. Dans ce qui suit, nous nous appuierons sur le texte $\mathrm{n}^{\circ} 7$ du volume XXXVI des Husserliana, qui date probablement de 19141915, donc d'une période immédiatement postérieure à la publication des Ideen ${ }^{22}$. Deux moments y sont essentiels pour nous. D'une part, Husserl affirme qu'un sujet effectif (wirklich) est requis pour la simple possibilité réelle (reale Möglichkeit) d'un monde, car " un sujet, seulement logiquement possible, $\mathrm{n}^{\prime}$ est pas un substrat pour des possibilités réelles ${ }^{23}$. La nature avant la conscience ne peut donc pas être comprise comme une nature pour une conscience seulement possible, puisque déjà sa simple possibilité réelle - et a fortiori son effectivité exige un sujet réel. Husserl voit ici une « thèse épistémologique générale importante »: "L'existence de tout être effectif [...] exige la coexistence nécessaire d'un sujet connaissant, respectivement d'un sujet capable de connaissance ${ }^{24}$. Cette thèse semble renforcer l'impasse. Mais il précise alors sa position : « ce sujet réellement existant [...] n'est pas exigé [...] pour tout le temps infini de l'existence du monde $»^{25}$. La solution proposée par Husserl au problème de la constitution phénoménologique d'un monde d'avant la conscience serait donc la suivante : «Un monde sans sujets qui l'expérimentent effectivement [...] $n^{\prime}$ est pensable que comme passé d'un monde avec de tels sujets ${ }^{26}$. Il est donc bien possible de rendre compte d'un "monde matériel en tant que premier cycle (Unterstufe) et première étape de la durée du monde », car un sujet actuel permet de "construire en arrière [...] les étapes du monde précédentes $»^{27}$. Une nature sans conscience ne

\footnotetext{
${ }^{22}$ Emmanuel Alloa a récemment discuté le problème d'une existence du monde «sans nous » chez Husserl dans «Le monde existe-t-il sans nous ? Le perspectivisme selon Husserl. Présentation ", in Philosophie, n 131, septembre 2016, p. 3-19. Cette présentation est suivie de deux traductions de Husserliana XXXIX, Beilage XLII, p. 509-513 (trad. p. 20-23) et de Husserliana XXXIX, texte $\mathrm{n}^{\circ} 57, \S 4$, p. 650-666 (trad. p. 24-35).

${ }^{23}$ E. Husserl, Transzendentaler Idealismus. Texte aus dem Nachlass (1908-1921), R. D. Rollinger et R. Sowa (éd.), Husserliana XXXVI, Dordrecht, Springer, 2003, p. 139.

${ }^{24}$ Ibid., p. 140, nous soulignons.

${ }^{25}$ Ibid., p. 140.

${ }^{26}$ Ibid., p. 144, nous soulignons. László Tengelyi a souligné cette réponse husserlienne et il la comprend comme " un dépassement en même temps de l'idéalisme et du réalisme ainsi que du subjectivisme et du naturalisme ». Voir Welt und Unendlichkeit. Zum Problem phänomenologischer Metaphysik, Fribourg et Munich, Verlag Karl Alber, 2014, p. 210-213, citation p. 212.

${ }^{27}$ Ibid., p. 141. Alloa évoque le rôle que Husserl semble accorder aux actes constituants des animaux pré-historiques. Ibid., p. 15. Mais même si l'on admettait les animaux pré-historiques en tant que partie de la communauté constituante, le problème ne serait que décalé vers le champ de la nature avant le surgissement de ces animaux.
} 
pourrait donc être constituée phénoménologiquement que comme le passé de la conscience actuelle et effective ${ }^{28}$.

\section{Ricour - la trace comme effet-signe entre causalité objective et signification subjective}

Approfondissons cette idée husserlienne en nous tournant vers la conception ricœurienne de la trace. L'une des thèses centrales de Ricœur, en effet, est que la dimension aporétique du temps - écartelé entre un temps subjectif et un temps objectif $-n^{\prime}$ est pas résorbable dans une conception unifiée de la temporalité, et ne peut trouver de cohérence qu'à travers des médiations poétiques et pratiques dont la trace est justement une instance essentielle. "La trace », dit ainsi Ricœur dans le troisième volume de Temps et récit, " est un effet-signe ${ }^{29}$. Effet d'une causalité objective dans le développement de la nature, elle est signe d'une antériorité et exige d'être interprétée pour avoir du sens pour nous. En tant qu'effet, elle s'insère dans un temps objectif de la nature ; en tant que signe, elle nous place devant un problème d'interprétation. Certes, confrontée à l'aporicité du temps, cette réponse implique que la fonction objective d'effet naturel ne peut jamais être entièrement détachée de la fonction subjective significative; mais elle peut être visée en tant que dimension abstractive limite du caractère double et tensionnel du temps. En appliquant une telle approche à l'idée husserlienne d'une constitution rétrospective d'une nature d'avant la conscience, nous pouvons donc dire : la nature avant la conscience est rétrospectivement constituée en tant que cause de l'effet qui en est actuellement la trace devant nous, même si cette nature en tant qu'étape d'un temps seulement objectif ne peut être pensée que comme dimension abstractive limite de notre passé subjectif.

Ces réflexions sur Husserl et Ricœur donnent ainsi une réponse à la question qu'annonçait la formule: "la nature derrière nous ». Mais qu'en est-il de la nature "en nous », qui, elle aussi, n'est pas un phénomène « devant nous »? Nous nous tournerons maintenant vers Levinas pour esquisser une réponse.

\section{Levinas - la nature en nous dans la sénescence}

Dans Autrement qu'être ou au-delà de l'essence, Emmanuel Levinas évoque à maintes reprises une "passivité [...] plus passive [...] que

\footnotetext{
${ }^{28}$ Cette réponse temporelle ressemble à la réponse donnée par Schelling dans les Âges $d u$ monde, où il comprend la nature comme l'âge du monde passé.

${ }^{29}$ P. Ricœur, Temps et récit. 3 : Le temps raconté, Paris, Seuil, 1985, p. 219.
} 
toute passivité corrélative du volontaire $»^{30}$. Cette profonde passivité est aussi bien liée à ma corporéité vivante qu'à la temporalité : «La dolence de la douleur, la maladie ou la malignité du mal, et, à l'état pur, la patience même de la corporéité, la peine du travail et du vieillissement - sont $l^{\prime}$ adversité même, le contre soi en soi $»^{31}$. Plus passives que la passivité qui est le pur contraire de l'activité sont les expériences de la douleur, de la maladie, de la patience corporelle, de la peine du travail et du vieillissement ; et ces expériences manifestent une temporalité particulière : "Le temps se passe », en se passant il se passe de moi, et «[c]ette synthèse qui patiemment se fait [...] est vieillissement ${ }^{32}$. Ce qui est ici en jeu, ce n'est donc pas seulement la synthèse des rétentions et des protentions qui s'effectuerait sans l'activité du sujet, mais bien des synthèses qui sont vieillissement dans la mesure où elles impliquent un échappement essentiel qui affecte et mine constamment la conscience de soi. Husserl a certes vu, dit Levinas, la passivité des synthèses du temps, mais à cette passivité s'ajoute ici cette passivité plus profonde qui est sénescence, une « sénescence pardelà la récupération de la mémoire ${ }^{33}$. Certes, Levinas ne présente pas la sénescence dans le cadre d'une philosophie de la nature; mais sa temporalité propre n'en atteste pas moins une nature en nous, expérimentée en tant qu'altérité d'une conscience qui tente en vain de maîtriser le temps par la présence du pour soi et par la remémoration lucide. Dans la temporalité la plus profonde s'effectue alors un retrait continu, et ce faisant une nouvelle figure de la nature : en marge de la nature en tant que passé plus haut dégagée avec Husserl et Ricœur, elle ne serait plus ni une nature en tant que phénomène devant nous, ni une nature en tant que concept spéculatif, mais une nature présente en nous en tant que retrait. Ajoutons du reste que, pour Levinas, cette naturalité de la conscience constitue la condition pour qu'un sujet « séparé » et « jouissant » puisse être touché par l'appel d'autrui :

Sous les espèces de la corporéité s'unissent les traits que l'ont vient d'énumérer : pour l'autre, malgré soi, à partir de soi; la peine du travail dans la patience du vieillissement, dans le devoir de donner à l'autre jusqu'au pain de sa bouche et du manteau de ses épaules. [...] Donner, êtrepour-l'autre, malgré soi, mais en interrompant le pour-soi, c'est arracher le pain à sa bouche, nourrir la faim de l'autre de mon propre jeûne ${ }^{34}$.

\footnotetext{
${ }^{30}$ E. Levinas, Autrement qu'être ou au-delà de l'essence, La Haye, Martinus Nijhoff, 1978, Kluwer, Le Livre de Poche, p. 87.

${ }^{31}$ Ibid., p. 87.

32 Ibid., p. 88, nous soulignons « est ».

${ }^{33}$ Ibid., p. 88.

${ }^{34}$ Ibid., p. 93, 94.
} 
Aussi est-ce l'altérité de la nature en moi qui rend possible l'êtrepour-l'autre ; la faim, la douleur, la fatigue en moi me laissent être touché par autrui. La naturalité en nous n'est alors pas le contraire de l'éthique, mais bien plutôt sa condition.

\section{Heidegger - le «Herstellen" de la terre dans le "Aufstellen" d'un monde}

La dernière figure phénoménologique que nous souhaitons évoquer est celle de la «terre», telle qu'elle se trouve développée par Heidegger dans « L'origine de l'œuvre d'art »35. Cette notion, devenue problématique depuis la publication des Cahiers noirs, exigerait une discussion détaillée que nous ne saurions mener ici, et nous nous contenterons d'en souligner un trait important pour notre propos.

L'un des concepts centraux de la pensée heideggérienne de la fin des années vingt est celui de " projet du monde »(Weltentwurf), lié à l'ekstase temporelle de l'avenir. Au milieu des années trente, Heidegger utilise encore le thème du projet, mais donne de plus en plus à l'ouverture $\mathrm{d}^{\prime}$ un monde une dimension événementiale. Néanmoins, l'ouverture du monde reste essentiellement liée à l'avenir, en ce sens qu'elle ouvre un champ significatif à l'intérieur duquel les différents étants reçoivent une signification particulière. Or dans les analyses qu'il consacre à l'œuvre d'art dans les conférences de 1936, Heidegger développe l'idée d'une complémentarité entre un Aufstellen d'un monde et un Herstellen de la terre : "Installant un monde, l'œuvre fait venir la terre (Indem das Werk eine Welt aufstellt, stellt es die Erde her) ${ }^{36}$. Et ce n'est que par contraste avec l'installation d'un monde par l'œuvre que la terre peut devenir phénomène :

Dans sa constance, l'œuvre bâtie tient tête à la tempête passant au-dessus d'elle, démontrant ainsi la tempête elle-même dans toute sa violence. L'éclat et la lumière de sa pierre, qu'apparemment elle ne tient que par la grâce du soleil, font ressortir la clarté du jour, l'immensité du ciel, les ténèbres de la nuit. Sa sûre émergence rend ainsi visible l'espace invisible de l'air. La rigidité inébranlable de l'œuvre fait contraste avec la houle des flots de la mer, faisant apparaître par son calme, le déchainement de l'eau. L'arbre et l'herbe, l'aigle et le taureau, le serpent et la cigale ne trouvent qu'ainsi leur figure d'évidence, apparaissant comme ce qu'ils sont ${ }^{37}$.

\footnotetext{
${ }^{35}$ Voir M. Heidegger, «Der Ursprung des Kunstwerkes (1935/36)», in Holzwege, F-W. von Herrmann (éd.), Gesamtausgabe vol.5, Francfort-sur-le-Main, Vittorio Klostermann, p. 1-74 («L'origine de l'œuvre d'art», in Chemins qui ne mènent nulle part, trad. W. Brokmeier, Paris, Gallimard, 1962, p. 13-98).

${ }^{36}$ Ibid., p. 32 (p. 49 sq.).

${ }^{37}$ Ibid., p. 28 (p. 44).
} 
Soulignons trois aspects importants d'une telle approche pour notre propos.

D'une part, ce n'est qu'à travers l'installation d'un monde que la terre devient phénomène, car elle se montre en tant que terre sous la condition d'un monde installé, «dans l'ouvert du monde de $l^{\prime} œ u v r e »^{38}$. Mais d'autre part, cette apparition va de pair avec une réserve : "Ouverte dans le clair de son être, la terre n'apparaît comme elle-même que là où elle est gardée et sauvegardée en tant que l'indécelable par l'essence, qui se retire devant tout décel, c'est-à-dire qui se retient en constante réserve ${ }^{39}$. Enfin, ce double aspect de phénomène et de réserve se concrétise en un moment de résistance: "C'est dans la terre, en tant que celle qui essentiellement se réserve, que l'ouverture de l'ouvert rencontre sa suprême résistance $»^{40}$. En ce sens, la terre est ce qui ne devient phénomène qu'en tant que résistant à l'ouverture du monde, et sa phénoménalisation est une phénoménalisation en tant que réserve et retrait. C'est l'ouverture du monde, avec son orientation vers l'avenir, qui rend possible une certaine phénoménalisation de la terre.

\section{Esquisse d'une conception temporelle de la nature}

Nous venons d'ouvrir quatre perspectives phénoménologiques sur la nature qui, attentives à certaines figures du retrait dans la phénoménalité même, neutralisent l'alternative entre, d'une part, une phénoménalité pleinement transparente et, d'autre part, une inaccessibilité phénoménale qui ne serait surmontable que par la spéculation. Le danger n'est-il pas toutefois, en convoquant rhapsodiquement des penseurs aussi différents que Husserl, Heidegger, Ricœur et Levinas, de faire preuve d'un éclectisme douteux? C'est précisément ce que permettrait d'éviter une conception temporelle de la nature qui, absente des textes que nous avons jusqu'ici mobilisés, ne serait pas faire écho à la pensée de Schelling dans Les âges $d u$ monde ${ }^{41}$.

Husserl et Ricœur, en effet, nous ont initiés à une nature irréductible à un phénomène actuellement "devant nous » car également située « derrière nous » et précédant la conscience phénoménologique elle-

\footnotetext{
${ }^{38}$ Ibid., p. 32 (p. 49).

39 Ibid., p. 33 (p. 50 s.).

${ }^{40}$ Ibid., p. 57 (p. 78, nous soulignons).

${ }^{41}$ F. W. J. Schelling, Die Weltalter. Fragmente. In den Urfassungen von 1811 und 1813, M. Schröter (éd.), Munich, Biederstein Verlag et Leibniz Verlag, 1946 (Les âges du monde. Fragments dans les premières versions de 1811 et 1813, trad. P. David, Paris, PUF, «Épiméthée », 2014).
} 
même. Plus précisément: la notion de trace en tant qu'effet-signe introduite par Ricœur permettrait de nuancer la thèse husserlienne selon laquelle la nature "devant nous » rendrait possible la constitution rétrospective d'une nature qui, "derrière nous », serait à comprendre comme notre passé. La notion d'objectivité naturelle ne serait alors qu'une abstraction limite de la double nature subjective et objective du temps, et l'assimilation et l'exploration de ce passé propre à la nature "derrière nous " dépendraient toujours déjà de notre interprétation actuelle de la causalité objective et naturelle.

De leur côté, les analyses de Levinas nous permettent de rendre compte d'une nature " en nous ", éprouvée comme le retrait propre à l'intériorité d'une conscience de soi s'échappant constamment dans les figures de la sénescence - un présent de la conscience par conséquent, toujours déjà sapée par la nature comme modalité intérieure de l'altérité mettant en cause la plénitude de son auto-transparence et limitant ses facultés de remémoration. La nature se manifeste ici sous la forme d'un présent qui nous échappe.

La complémentarité, enfin, de l'Aufstellen d'un monde et du Herstellen de la terre chez Heidegger fait alors signe vers un dernier moment temporel d'une phénoménologie de la nature. L'installation d'un monde est liée à l'avenir, en ceci qu'elle ouvre un champ significatif au sein duquel la terre peut, par contraste, devenir phénomène. Mais la terre elle-même n'apparaît que comme ce qui se réserve et résiste à l'installation d'un monde. Or ne serait-il pas possible de lier cette découverte à l'interprétation de la nature objective mise au jour par Husserl et Ricœur? D’une part, nous ne saisissons bien la nature comme un «devant » et un « derrière » nous qu'à partir d'une ouverture ou d'une installation d'un monde intrinsèquement liée à un cadre scientifique historiquement circonscrit. Mais d'autre part, et comme y insiste Heidegger, la nature elle-même ne saurait se résorber dans une telle interprétation : elle ne devient au contraire phénomène que par contraste et ne se montre qu'en se retirant de et en résistant à toute installation d'un monde.

Cette esquisse d'une phénoménologie de la nature fait ainsi signe vers une circularité non-vicieuse, herméneutique et essentiellement temporelle, au sein de laquelle trois moments apparaissent chaque fois à la lumière des autres: l'interprétation d'une nature objective « devant» ainsi que « derrière nous » par la voie d'une constitution rétrospective d'un passé de la conscience avant la conscience, l'expérience actuelle d'un retrait intérieur à la conscience phénoménologisante elle-même, et finalement la résistance d'une nature contre toute inscription dans un monde particulier qui servirait, à une époque 
donnée, de cadre interprétatif. Ces trois moments, dégagés à partir de quatre cadres théoriques bien différents, trouveraient ainsi leur unité dans une phénoménologie herméneutique de la nature prenant essentiellement une forme temporelle. 